\title{
EL ECOSISTEMA DE EMPRENDIMIENTOS TECNOLÓGICOS EN LA CIUDAD DE CÓRDOBA. PRINCIPALES CARACTERÍSTICAS Y CONTRIBUCIONES SOBRE LA ECONOMÍA LOCAL
}

\section{The ecosystem of technological ventures in the city of Córdoba. Main characteristics and contributions on the local economy}

\section{Luciano Crisafulli', Daniel Parisi²}

Recibido: 20/09/2021 - Aceptado: 10/11/2021

\section{RESUMEN:}

El objetivo de este artículo es caracterizar algunos aspectos del ecosistema de emprendimientos tecnológicos de la Ciudad de Córdoba a partir de un relevamiento realizado a 167 emprendimientos radicados en dicha ciudad. El enfoque se orienta al análisis del perfil general de estos emprendimientos; las principales características de los equipos emprendedores; el empleo y facturación generada; las fuentes del financiamiento obtenido; los principales obstáculos para su crecimiento; como así también su posición relativa con otros ecosistemas.

\section{ABSTRACT}

The purpose of this article is to describe a series of aspects of the tech startup ecosystem in Cordoba City, from a survey conducted on 167 startups settled in said city. The focus is oriented to the analysis of the general profile of these startups, the main characteristics of the startups teams, their employment and generated income, the sources of funds received, the main obstacles in their growth, as well as their relative position with other ecosystems.

PALABRAS CLAVE: emprendimientos tecnológicos, emprendimiento, innovación, desarrollo económico.

JEL: L26, M13, O10, O31

KEYWORDS: technological ventures, entrepreneur, innovation, economic development. JEL: L26, M13, O10, O31

\footnotetext{
${ }^{1}$ Profesor Asistente en Facultad de Ciencias Económicas, Universidad Nacional de Córdoba. Profesor de Posgrado en Universidad Blas Pascal. Director de Innovación en Municipalidad de Córdoba. lcrisafulli@eco.uncor.edu.

2 Profesor Asistente en Facultad de Ciencias Económicas, Universidad Nacional de Córdoba. Profesor en Universidad Siglo 21. danielparisi@unc.edu.ar.
} 


\section{Introducción}

Durante los últimos años ha cobrado especial interés el estudio sobre los ecosistemas de emprendimientos tecnológicos y el impacto de estos sobre el desarrollo económico de las ciudades. Ello obedece a que este tipo de emprendimientos pertenece a uno de los sectores más dinámicos de la economía, a saber, el sector de las tecnologías de la información y comunicación (TIC).

Los emprendimientos tecnológicos son señalados a nivel mundial como agentes de transformación productiva, ya que cumplen un rol diferencial con relación a las empresas más tradicionales. Los emprendimientos tecnológicos funcionan sobre la base de una tecnología disruptiva y un modelo de negocio exponencial, lo cual los convierte en organizaciones de alto valor agregado y productividad; ágiles y adaptables frente a cambios en el entorno; $y$, con potencial de rápido crecimiento en el tiempo.

Este tipo de organizaciones cobran un especial interés en las economías latinoamericanas, las cuales se caracterizan por contar con estructuras productivas poco dinámicas y con bajos niveles de valor agregado y productividad. Los emprendimientos tecnológicos, tienen el potencial de complejizar el entramado productivo dotando de mayor tecnología e innovación a toda la matriz productiva, elevando el nivel de productividad e ingreso de la economía.

En Argentina, ciudades como Ciudad Autónoma de Buenos Aires (CABA), Córdoba, Rosario y Mendoza se muestran como posibles entornos donde propiciar la proliferación de emprendimientos tecnológicos que contribuyan con la transformación productiva de la economía. Ello responde principalmente a dos factores: a) la significativa dotación relativa de capital humano existente en dichas ciudades; $y, b)$ la existencia de un nutrido entramado institucional compuesto por universidades y organizaciones de apoyo emprendedor como fondos de capital de riesgo, aceleradoras, incubadoras, fundaciones, asociaciones de emprendedores, entre otros.

Desde el Laboratorio de Innovación Pública y Social de la Ciudad de Córdoba (CorLab), se implementó en el segundo semestre del año 2020 una iniciativa denominada "Startup Córdoba Monitor". La misma consiste en una actividad que releva información sobre los emprendimientos tecnológicos radicados en la Ciudad de Córdoba. El objetivo de dicha iniciativa es contar con una radiografía del ecosistema local que permita conocer su composición, diagnosticar su estado actual e identificar las principales características del sector para que, organizaciones públicas, privadas y del tercer sector, puedan contar con información de primera fuente para el proceso de toma de decisiones que impliquen acciones de mejora del ecosistema.

El presente artículo busca sistematizar la información relevada por dicho Monitor ante la necesidad de contar con estudios locales que den cuenta del aporte que hacen los emprendimientos tecnológicos a la economía local. Para ello, en una primera sección se presentan algunas definiciones y conceptualizaciones de los emprendimientos tecnológicos y de sus impactos en la economía de un territorio o región. Luego, en segundo lugar, se presenta la importancia del entramado institucional para el desarrollo de emprendimientos tecnológicos. En tercer lugar, se muestran algunas características del entramado institucional del ecosistema local. En cuarto lugar, se revelan los resultados del perfil de los emprendimientos tecnológicos de la Ciudad de Córdoba, allí se presentan características tales como los rubros presentes, fuentes de financiamiento, modelos de negocios elegidos, tecnologías utilizadas, empleos generados, entre otras. Por último, con la finalidad de realizar un análisis comparativo, se analizan diferencias y similitudes del perfil de los emprendimientos tecnológicos de Córdoba con los de España y Alemania.

\section{El impacto de los emprendimientos tec- nológicos en la economía}

En la historia del pensamiento económico pueden encontrarse diferentes teorías que explican las claves del crecimiento económico. Muchas de ellas coinciden en el factor de la innovación como fuente promotora del crecimiento.

A mediados del siglo 20, el economista austríaco Joseph Schumpeter atribuía a la in- 
novación introducida en los procesos de producción, como causante del impulso del crecimiento de una economía. En su libro "Capitalismo, Socialismo y Democracia" (1942), argumentaba que la innovación consiste en un proceso de "destrucción creadora" que modifica la estructura económica, al destruir lo antiguo para crear elementos nuevos más eficientes y generadores de mayor bienestar para la sociedad.

A su vez, cuando se implementa una innovación, surgen externalidades positivas para la economía, ya que se minimizan costos y recursos y/o se genera mayor valor agregado a lo producido. De esta manera, la innovación contribuye a mejorar la satisfacción del usuario, aumentar la rentabilidad del oferente y eficientizar a los competidores, generando un funcionamiento dinámico en la economía.

La aplicación de modelos endógenos de crecimiento económico argumenta la importancia y los efectos derrames del conocimiento y de las nuevas tecnologías (Varga y Schalk, 2004). Bajo esta concepción, las economías comienzan a desarrollar ecosistemas emprendedores dinámicos generadores de cambios positivos disruptivos, como ocurre en California (EEUU) e Israel, por ejemplo.

Estos ecosistemas dinámicos están compuestos de emprendimientos que surgen como iniciativas empresariales impulsadas por equipos de emprendedores con alto nivel de formación, que identifican una oportunidad de negocio regional o global y que tienen un alto potencial de crecimiento apalancado principalmente en las tecnologías exponenciales.
Para un territorio, la aglomeración de este tipo de emprendimientos intensivos en tecnología, son clave para dotar de una mayor complejidad, dinamismo y valor agregado a su matriz productiva. En otras palabras, en volumen, estas organizaciones realizan un importante aporte sobre indicadores socioeconómicos como nivel de productividad e ingreso, teniendo un alto potencial de contribución sobre el desarrollo económico local.

Algunas de las principales características distintivas de los emprendimientos dinámicos son las siguientes: a) alcanzan altos niveles de facturación y obtienen importantes volúmenes de inversión privada durante los primeros años de vida; b) exhiben un crecimiento exponencial a lo largo del tiempo; c) poseen una clara voluntad de acumulación de tecnología y conocimiento; $y, d$ ) presentan un alto grado de diferenciación e innovación.

Estas organizaciones son conocidas también como "emprendimientos gacelas", una metáfora del reino animal planteada por David Birch (1987), la cual intenta reflejar el ciclo de vida de las empresas y su impacto sobre la creación de riqueza y puestos de trabajo. Según este autor, al inicio los emprendimientos son como los ratones, hay muchos, pero no crecen y entonces no impactan significativamente en la creación de riqueza y nuevos empleos. Luego, aparecen las gacelas, que son las empresas jóvenes de rápido crecimiento. Y, finalmente, están las grandes empresas, que se mueven lento y generan muy pocos nuevos puestos de trabajo, por eso las asemeja a los elefantes.

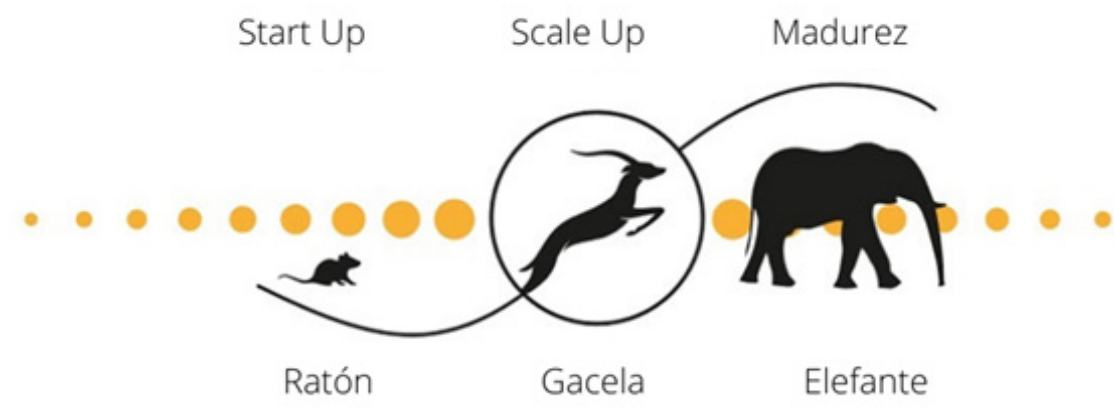

Figura 1: El Ciclo de las compañías. Fuente: Elaboración propia en base a Birch (1987) 
Como se observa, existen diferentes acepciones sobre los emprendimientos tecnológicos, sin embargo, todas ellas tienen como elemento común la posibilidad de escalar rápidamente y generar innovación. Teniendo en cuenta esto, podemos definir a un emprendimiento tecnológico, o startup, como una empresa emergente que, a través del uso de tecnología, ofrece productos o servicios innovadores con un modelo de negocio de crecimiento rápido y escalable.

Teniendo en consideración entonces la importancia que tienen los emprendimientos tecnológicos surge la pregunta acerca de cómo promover en un territorio el surgimiento o crecimiento de estos. En este sentido, Daniel Isenberg (2011), en base a un análisis del modelo de Israel, definió que debe implementarse una "estrategia de ecosistema emprendedor" para el desarrollo económico. La estrategia consiste en agrupar seis factores: a) mercado; b) políticas; c) financiamiento; d) apoyos; e) cultura; $y$, f) capital humano.

De acuerdo con un estudio de Kantis y Angelelli (2020), existen factores impulsores para los emprendimientos como: la motivación de abordar una oportunidad de mercado; los recursos y financiamientos disponibles; $y$, los enlaces, vinculaciones y alianzas que puedan generarse en el territorio. Sin embargo, a nivel macroeconómico, influyen también los factores políticos, sociales, culturales y tecnológicos.

Un complemento a estas conclusiones son las propuestas por Patiño y García de León (2018), quienes plantean factores que impulsan, en términos generales, el desarrollo empresarial: a) inversión pública en infraestructura: incluye desde el acceso a tecnología hasta caminos y vías para el movimiento de los productos; b) facilidades fiscales: incentivos que el gobierno puede otorgar para fomentar cierto sector o actividad; c) inversión en innovación y educación: formación del talento local en conocimientos que contribuyan a la actividad emprendedora; d) Estado de derecho y seguridad: generar un clima de negocio ágil y sin incertidumbres legales en el que se respeten los derechos de propiedad y que se puedan apropiar de sus innovaciones.
Un emprendimiento tecnológico, como cualquier empresa, no solo depende del talento de sus socios fundadores sino también de su entorno o ecosistema. Esto es lo que se llama ventaja comparativa: que la ubicación sea mejor que otras ubicaciones en cuanto a la existencia de apoyo a la competitividad de las empresas. En tales aspectos, el sector público tiene un desempeño fundamental.

En América Latina, particularmente desde la segunda década del siglo 21 , el fenómeno de los emprendimientos tecnológicos ha atraído una atención creciente por parte de medios de comunicación, académicos, inversionistas privados y de formuladores de políticas. Entre 2012 y 2015 las políticas de apoyo a los emprendimientos tecnológicos se han introducido en varios países, como por ejemplo Chile, Colombia, México y Perú (OCDE, 2013), pero también en Uruguay, Argentina y Panamá, entre otros (OCDE, 2016). Estas políticas, a diferencia de otras intervenciones más tradicionales de apoyo a la competitividad empresarial, han evolucionado rápidamente, y en pocos años se han generado cambios importantes en su lógica, enfoque y estructura. Un ejemplo claro de ello es la aparición de programas de inversión pública en emprendimientos tecnológicos a través de modalidades de coinversión con el sector privado (matching-fund).

Los emprendimientos tecnológicos tienen un rol fundamental en el desarrollo económico de un territorio, ya sea desde el punto de vista de la generación de empleo de calidad y valor agregado como así también, por la generación de innovaciones que impactan positivamente sobre el bienestar social. Asimismo, estos emprendimientos tienen una gran relevancia productiva, pues sus innovaciones tecnológicas afectan transversalmente a una multitud de industrias y sectores. En definitiva, el desarrollo y consolidación de emprendimientos tecnológicos en el entramado productivo genera múltiples impactos sobre la economía de un territorio. De allí la importancia del apoyo a estos ecosistemas de "emprendimientos tecnológicos", que son verdaderamente agentes de cambios productivos, económicos y sociales. 
III. La importancia del entramado institucional para el desarrollo de emprendimientos tecnológicos

Se entiende como ecosistema al sistema en el cual interactúan organizaciones, individuos y otros elementos tangibles e intangibles. El modelo más antiguo y utilizado es el denominado "triple hélice" de Etzkowitz y Leydesdorff (2000). El mismo se basa en entender al ecosistema como la interacción entre los actores del sector privado, gobierno y academia. Más adelante, se incorpora al modelo una cuarta hélice que incluye a la sociedad civil.

En la actualidad, esta clasificación resulta aún incompleta al momento de profundizar y comprender las dinámicas complejas que suceden dentro de los ecosistemas modernos, ya que se centran en una clasificación sectorial más que en las funciones mismas que se cumplen dentro del ecosistema. Por ello, hay una evolución donde se destacan 5 hélices, o más, y por lo tanto ya se denomina la N-tuple hélice.

Como señala Castillo-Vergara (2020), "la quíntuple hélice representa una interacción colectiva, un intercambio de conocimiento que incluye cinco subsistemas o hélices: (1) Sistema Educativo; (2) Sistema Económico; (3) Entorno Natural; (4) el Público basado en los medios de comunicación y en la cultura y/o sociedad civil y (5) el sistema político".

Para comprender en un sentido más amplio a los ecosistemas, se tornó útil utilizar herramientas de mapeos que permiten identificar los actores integrantes que van a interactuar y generar sinergias. Estos mapeos llevaron a una nueva concepción de ecosistema: el modelo basado en actores que se compone de seis pilares y seis tipos de actores. Los seis pilares son: a) identificar; b) capacitar; c) conectar y sostener; d) financiar; e) habilitar; $y$, f) celebrar. Mientras que los seis tipos de actores que interactúan sobre esos pilares son: a) Articuladores: son los que aseguran la creación de espacios y plataformas propicias para que los diferentes actores no solo "hablen" entre sí, sino que colaboren activamente en iniciativas conjuntas. b) Habilitadores: proveen recursos al ecosistema para apoyar la generación y el desarrollo de nuevos emprendimientos tecnológicos. Los habilitadores son todos aquellos agentes que proveen herramientas y recursos como formación, talento, consultoría, recursos financieros, infraestructura y espacios, entre otros. Éstos permiten al ecosistema incrementar el nivel de recursos disponibles para que tanto actores como emprendedores puedan reducir las barreras de entrada que el mismo ecosistema y/o mercado tienen naturalmente. Independientemente de las inversiones monetarias, los habilitadores aportan recursos tan estratégicos como los fondos en sí.

c) Vinculadores: Conectan emprendedores, empresas y actores dentro del ecosistema para crear nuevas relaciones. Dichas relaciones institucionales, fomentan la colaboración y la compartición de conocimiento sobre el mercado y mejores prácticas, y permiten enfrentar oportunidades y problemas de manera conjunta, fortaleciendo la posición de estas instituciones.

d) Generadores de conocimiento: Instituciones públicas y privadas que generan nuevo conocimiento, que a su vez pueden impulsar la creación de nuevos proyectos, tecnologías, innovación y emprendimientos de alto impacto. e) Promotores: Divulgan y promueven el emprendimiento de alto impacto, local e internacionalmente, para apoyar la escalabilidad de los proyectos y fomentar la cultura de emprendimiento e innovación.

f) Comunidades: Organizaciones civiles formales o no que comparten conocimiento, colaboran y dan dinamismo al ecosistema de emprendimiento de alto impacto. Las Comunidades, si bien pertenecen a la sociedad civil, generalmente en su nacimiento son "no organizadas", ya que nacen no como instituciones formales, sino como entes con el propósito puro de compartir el conocimiento, de apoyarse mutuamente y de enriquecerse a partir de intereses únicos comunes. Son organizaciones que persisten más allá de las políticas públicas de los gobiernos de turno, por lo que son actores del ecosistema que le dan sostenibilidad y continuidad al mismo.

La Ewing Marion Kauffman Foundation (2014), 
en línea con los ecosistemas emprendedores basados en actores, define el rol que estos juegan en el ecosistema. Sostiene que el ecosistema debe contener: a) emprendedores que aspiran a crear y desarrollar nuevos negocios; b) talento que puede ayudar a las compañías a crecer; c) personas e instituciones con conocimiento y recursos para ayudar a emprendedores; d) individuos e instituciones que sean defensores de los emprendedores y el ecosistema; e) puntos de acceso al ecosistema para que cualquiera y todos participen; f) intersecciones que faciliten la interacción entre personas, ideas y recursos; g) historias que las personas cuentan sobre sí mismas y su ecosistema; h) cultura que es rica en capital social, colaboración, cooperación, confianza, reciprocidad y con enfoque en el bien común.

\section{El ecosistema de emprendimientos tecnológicos en la ciudad de Córdoba}

El apoyo a emprendimientos tecnológicos ha adquirido en los últimos años una importancia significativa en las principales ciudades del mundo. La justificación de ello radica en el gran impacto que los mismos generan sobre la economía local a través de diferentes vías. Como se señaló anteriormente, estos emprendimientos contribuyen con la dinamización de la matriz productiva local, no sólo por el alto nivel de productividad que presentan, sino también por la innovación, tecnología y agilidad que aportan al resto del entramado productivo. Además, el sector tecnológico brinda oportunidades laborales de calidad en forma directa e indirecta, con el potencial de favorecer el ascenso social de la ciudadanía a través del círculo virtuoso que implica la reasignación de recursos desde actividades menos productivas hacia actividades más sofisticadas.

La Ciudad de Córdoba cuenta con un ecosistema emprendedor tecnológico en donde diferentes organizaciones públicas, privadas y del tercer sector trabajan de forma mancomunada para apoyar el nacimiento y crecimiento de emprendimientos tecnológicos. EI relevamiento publicado por Crisafulli y Parisi (2020) muestra que, entre estas organizaciones, aparecen asociaciones empresarias y fundaciones de larga trayectoria que desarrollan en forma permanente diversas actividades que contribuyen con la generación de comunidades conformadas por emprendedores, empresarios, inversores, referentes sociales y académicos.

También es significativo el rol que desempeñan las incubadoras de emprendimientos, la mayoría de ellas dependientes de Universidades, que acompañan a los emprendedores a través de actividades de capacitación, vinculación y asistencia técnica. Estas acciones favorecen la transferencia de conocimiento desde el sector académico al sector productivo en el ecosistema local.

La Ciudad de Córdoba cuenta con instituciones de capital de riesgo, algunas de ellas reconocidas a nivel internacional, que invierten en emprendimientos tecnológicos en diferentes estadíos.

Finalmente, el Estado también cumple un rol activo a través de la implementación de políticas públicas nacionales, provinciales y locales que impulsan al sector.

Esta densidad institucional genera un entorno favorable para el nacimiento y crecimiento de emprendimientos tecnológicos, generando un impacto directo sobre la economía local.

A partir de los resultados del Startup Córdoba Monitor publicados en la plataforma de gobierno abierto de la Municipalidad de Córdoba, se puede conocer que el $64 \%$ de los emprendimientos tecnológicos de la ciudad obtuvo algún tipo de apoyo de parte de alguna institución del ecosistema. De estos emprendimientos, el $48,5 \%$ señaló que obtuvo apoyo por más de una institución.

En cuanto al financiamiento obtenido en el ecosistema, el $75 \%$ de los emprendimientos tecnológicos de la Ciudad de Córdoba logró algún tipo de apoyo económico. De los que accedieron a financiamiento, la primera fuente fue la de programas públicos en un $42,4 \%$, luego préstamos de familiares y amigos en un $30,4 \%$ y en tercera posición inversores ángeles con el 21,7\%. 
Tabla 1. Fuentes de financiamiento

\begin{tabular}{lc}
\hline Fuente & $\mathbf{\%}$ \\
\hline Programas de gobierno & $42,4 \%$ \\
Préstamos familiares y amigos & $30,4 \%$ \\
Inversores ángeles & $21,7 \%$ \\
Préstamos bancarios & $14,1 \%$ \\
Venture capital & $7,6 \%$ \\
Corporate venture & $6,5 \%$ \\
Crowdfunding & $2,2 \%$ \\
Otro & $1,1 \%$
\end{tabular}

Fuente: Elaboración propia en base a Startup Córdoba Monitor

Solo el $28,5 \%$ de los emprendimientos tecnológicos han recibido en Córdoba inversión de capital de riesgo. De ese grupo, el 54,3\% recibió más de 100 mil dólares y un 14,3\% recibió más de 1 millón de dólares. Los emprendimientos tecnológicos que recibieron este capital de riesgo se caracterizan por estar consolidadas: el 78\% está exportando y el $79 \%$ cuenta con más de 8 empleados. Además, el 95\% recibió el apoyo de alguna institución a lo largo de su crecimiento.

Por último, con relación a los factores de crecimiento, se les consultó a los emprendimientos cuáles eran los más determinantes teniendo en cuenta las siguientes categorías: - Financiamiento: que incluye la oportunidad y facilidad de acceder a capital.

- Contexto macroeconómico: condiciones económicas externas a las empresas, pero que afectan su funcionamiento.

- Recursos humanos competentes: Posibilidad de acceder y mantener recursos humanos capacitados para las actividades que desarrolla el emprendimiento.

- Infraestructura tecnológica: Accesibilidad a tecnologías necesarias para validar o comercializar el producto o servicio brindado.

- Carga impositiva: Cantidad y monto de impuestos de las distintas jurisdicciones (nacionales, provinciales y municipales).

- Trámites gubernamentales: Conjunto de requerimientos para poder formalizar el emprendimiento.

- Instituciones de apoyo: Programas y servicios ofrecidos por instituciones con el objetivo de fortalecer habilidades, vínculos y cono- cimientos de los emprendedores que tienen dirigen un emprendimiento tecnológico.

En el relevamiento, el emprendimiento debía evaluar cada una de las categorías en una escala del 1 al 10, en donde los valores más altos representan los factores que más inciden en la evolución del emprendimiento.

Entre los factores de crecimiento considerados más importantes, predomina poder contar con recursos humanos competentes $(7,3)$ y con infraestructura tecnológica $(7,3)$, luego aparece el contexto macroeconómico $(6,9)$ y, en cuarto lugar, la carga impositiva $(6,2)$.

Dado que el promedio podría estar distorsionado por valores extremos, se analizó la cantidad de respuestas para cada puntaje (valor modal), dando los mismos resultados que recién con la excepción del financiamiento, que intercambia de lugar con el factor de carga impositiva.

Tabla 2. Factores de crecimiento

\begin{tabular}{lc}
\hline Factor & Promedio \\
\hline Recursos humanos & 7,3 \\
Infraestructura tecnológica & 7,3 \\
Contexto macroeconómico & 6,9 \\
Carga impositiva & 6,2 \\
Financiamiento & 6,0 \\
Trámites gubernamentales & 5,7 \\
Instituciones de apoyo & 5,6
\end{tabular}

Fuente: Elaboración propia en base a Startup Córdoba Monitor

\section{Los emprendimientos tecnológicos en la ciudad de Córdoba}

En esta sección se muestran los resultados del primer relevamiento de emprendimientos tecnológicos de la Ciudad de Córdoba realizado por el Laboratorio de Innovación Pública y Social de la Ciudad de Córdoba, CorLab, con el acompañamiento de las siguientes instituciones del ecosistema tecnológico local durante los meses de septiembre y octubre del año 2020. 
El estudio incluyó a 167 emprendimientos que cumplieron con los siguientes criterios:

- Estar ubicados en la Ciudad de Córdoba y alrededores;

- Se encuentran en etapa de comercialización o bien en validación con prototipo terminado; $y$,

- Ofrecen soluciones de base tecnológica.

A continuación, se presentan las principales características del perfil de los emprendimientos tecnológicos de la Ciudad de Córdoba.

\section{V.1 Perfil de los emprendimientos tecno- lógicos}

En cuanto a su oferta, sobresalen los emprendimientos tecnológicos que ofrecen exclusivamente productos y servicios de software, llegando a representar el 75,3\% del total. En un porcentaje inferior, el $24,7 \%$ ofrece una solución que incluye software y hardware.

En cuanto a las etapas de los emprendimientos relevados, el $67,1 \%$ se encuentra comercializando productos y/o servicios, mientras que el 32,9\% restante está en etapa de validación con prototipo terminado (algunos de ellos ya se encuentran formalizados).

En la Figura 2 y 3 se muestran los resultados sobre la formalización de los emprendimientos. El $76 \%$ se encuentra constituido formalmente, mientras que el $24 \%$ restante indicó que aún lo tiene pendiente. En cuanto a la forma jurídica elegida, la gran mayoría se distribuye entre SAS con el $40 \%$ y en monotributistas con el 31\%. En porcentajes inferiores se encuentran las SA, SRL y SH.
Figura 2: ¿Está formalizada?

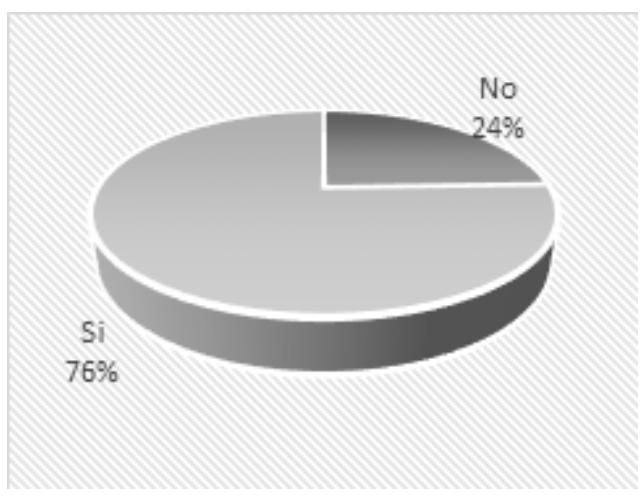

Fuente: Elaboración propia en base a Startup Córdoba Monitor

Respecto a la antigüedad de los emprendimientos, se observa que más de la mitad tiene menos de 3 años de actividad y tan solo el $19 \%$ supera los 5 años. La mayor proporción de los emprendimientos tecnológicos tienen entre 1 a 3 años. El apoyo público y privado al sector puede contribuir a que cada vez más compañías logren superar el famoso "valle de la muerte" para luego facilitar un crecimiento exponencial en los mismos.

En la Ciudad de Córdoba, como indica la Tabla 3, existe una importante diversificación de los sectores tecnológicos al que pertene-
Figura 3: Forma Jurídica

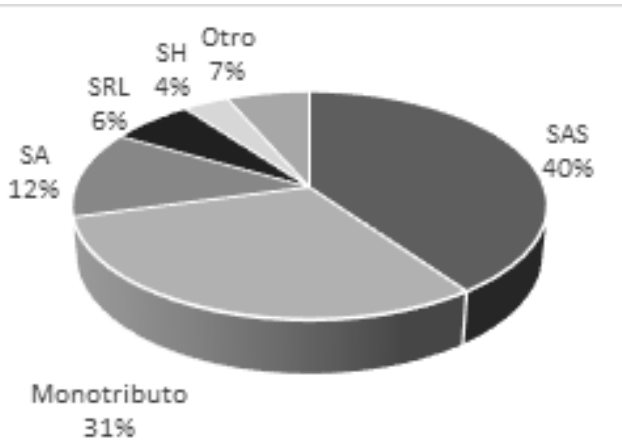

cen los emprendimientos tecnológicos, con más de 17 rubros presentes sin predominar ninguno en particular. Sin embargo, si se contemplan los 5 primeros, se encuentra que el $79,8 \%$ de los emprendimientos tecnológicos desarrollan productos o servicios en las verticales de Software factory, Comercio electrónico, Salud, Educación y Finanzas. Cabe mencionar que la suma de porcentajes de la Tabla 3 puede superar el $100 \%$ debido a que un emprendimiento tecnológico puede desarrollar y ofrecer productos vinculados a más de una vertical. 
Tabla 3. Vertical del emprendimiento

\begin{tabular}{lc}
\hline Vertical & $\%$ \\
\hline Software factory & $19.8 \%$ \\
Comercio Electrónico & $19.8 \%$ \\
Salud (E-health) & $16.2 \%$ \\
Educación (Ed-Tech) & $13.2 \%$ \\
Finanzas (Fintech) & $10.8 \%$ \\
Sustentabilidad & $9.6 \%$ \\
Marketing & $9.6 \%$ \\
Agro (Agtech) & $9.0 \%$ \\
Construcción (Proptech) & $7.2 \%$ \\
Transporte y movilidad & $6.6 \%$ \\
Logística & $6.0 \%$ \\
Redes sociales & $4.8 \%$ \\
Accesibilidad & $4.2 \%$ \\
RR.HH. & $3.6 \%$ \\
Videojuegos & $3.0 \%$ \\
Seguros (insurtech) & $3.0 \%$ \\
Seguridad & $2.4 \%$ \\
Otras industrias tecnológicas & $22.2 \%$
\end{tabular}

Fuente: Elaboración propia en base a Startup Córdoba Monitor

Entre las principales tecnologías bajo las cuales se apalancan los emprendimientos, en la Tabla 4 puede observarse que sobresale la tecnología de soluciones móviles. También son importantes las tecnologías de big data, nube e inteligencia artificial.

\section{Tabla 4. Tecnología utilizada}

\begin{tabular}{lc}
\hline Tecnología & \% \\
\hline Soluciones móviles & $48 \%$ \\
Big Data / Data Analytics & $31 \%$ \\
Computación en la nube & $28 \%$ \\
Inteligencia artificial & $28 \%$ \\
Internet de las cosas & $18 \%$ \\
Blockchain & $12 \%$ \\
Robótica & $9 \%$ \\
Realidad aumentada & $7 \%$ \\
Ciberseguridad & $7 \%$ \\
Impresión 3D & $6 \%$ \\
Simulación & $4 \%$ \\
Automatización & $4 \%$ \\
Otras & $15 \%$
\end{tabular}

Fuente: Elaboración propia en base a Startup Córdoba Monitor
Por último, en cuanto al modelo de negocio o fuente de monetización, el más elegido es el B2B (business to business) en el que sus clientes son otras empresas con el $53,7 \%$ del total. El modelo B2C (business to consumer) orientado a particulares asume el 37,2\% del total y el $26,2 \%$ orienta sus ventas tanto a empresas como a particulares (B2B2C). Además, existe un $22,6 \%$ de emprendimientos $B 2 G$ (business to government), es decir, aquellos que tienen como clientes los gobiernos. La suma de los porcentajes es superior al 100\% porque algunos emprendimientos cuentan con múltiples fuentes de monetización.

\section{V.2 Perfil de los emprendedores tecnológicos}

La cantidad de emprendedores fundadores de los emprendimientos tecnológicos relevados asciende a 534 en la Ciudad de Córdoba. Es decir, el promedio de fundadores por emprendimiento en el ecosistema local es de 3,2. Otro dato a considerar es que más del $60 \%$ son equipos formados por dos o tres integrantes.

En cuanto a la composición por género, sobresale que la gran mayoría de los fundadores son hombres, precisamente el $86,4 \%$, mientras que tan solo el $13,6 \%$ son mujeres. La brecha de género en el sector tecnológico de Córdoba es notable, tan solo el $2 \%$ de los emprendimientos son fundados íntegramente por mujeres, subiendo a $29 \%$ para el caso de equipos mixtos. Es necesario profundizar en programas que favorezcan y promuevan la participación de más mujeres en el ecosistema tecnológico.

Los equipos emprendedores muestran un importante nivel educativo. El $78 \%$ de los fundadores cuentan con estudios universitarios o de posgrado, mientras que tan solo un $11,6 \%$ de los fundadores cuentan con estudios secundarios. Es evidente que el nivel de estudios contribuye a la conformación de emprendimientos tecnológicos.

La edad promedio de los fundadores es de 34 años, donde tres cuartos de los emprendedores tienen más de 31 años. Solo un 6,4\% emprende con menos de 25 años y, en el otro extremo, aproximadamente un cuarto supera los 40 años. 


\section{V.3 Aporte de los emprendimientos tecno- lógicos a la economía local}

En lo que respecta al empleo directo que generan los emprendimientos tecnológicos en la Ciudad de Córdoba, dos de cada tres emprendimientos tienen empleados, mientras que el tercio restante es gestionado completamente por sus fundadores. El $41 \%$ tiene entre 1 y 3 empleados mientras que tan solo el $4.8 \%$ supera los 30 empleados. El número total de empleos generados se estima en 854 que, sumados a la cantidad de fundadores, asume el valor de 1.371 personas trabajando en emprendimientos tecnológicos en la Ciudad de Córdoba.

En cuanto a los niveles de facturación, del total de emprendimientos que ya cuentan con un producto final, el 15\% aún no logró facturar, mientras que aproximadamente un $49 \%$ solo facturó menos de 35 mil dólares anuales. Luego, solo el $16 \%$ de los emprendimientos que están comercializando superaron los U\$D 134 mil anuales de facturación. Del estudio se desprende que la facturación anual de los emprendimientos tecnológicos en la Ciudad de Córdoba es aproximadamente de U\$S 5.4 millones de dólares, un monto relativamente bajo si se tiene en cuenta el alto potencial de este tipo de organizaciones.

Dentro del grupo que más facturaron, se encuentra que el $87,5 \%$ de los emprendimientos ha logrado exportar, el $56 \%$ cuenta con más de 5 años de antigüedad y el $81 \%$ contó con el apoyo de alguna institución del ecosistema.

Del total de emprendimientos que se encuentran facturando, un $84 \%$ aumentó su facturación en el 2020 respecto al 2019 y un $27 \%$ logró que su facturación suba más del $100 \%$. De estos proyectos, un poco más de la mitad tiene una antigüedad de entre 1 a 3 años y cerca de dos tercios ya se encuentran exportando.

Respecto a los mercados en el que venden sus productos o servicios, la mayoría de los emprendimientos aún permanecen en el mercado nacional: el 89,2\% opera en Córdoba y el $78,5 \%$ en el mercado nacional. Solo el
$23,7 \%$ de los emprendimientos tecnológicos ya se encuentran en la etapa de internacionalización, la mayoría de éstos, 95,5\%, opera en mercados latinoamericanos, el $31,8 \%$ en el mercado europeo, el $22,7 \%$ en el mercado norteamericano y, finalmente, el 4,5\% logró vender a otras regiones como Asia. Estos datos revelan un bajo perfil global de los emprendimientos cordobeses, sin dudas un aspecto a tratar en el ecosistema.

Además de generar más de U\$S 5 millones de dólares de facturación anual y más de 1300 puestos de trabajo, se les consultó a los emprendimientos cuál era su consideración respecto al impacto de sus compañías en el entorno. El 68\% considera que ha logrado desarrollar una solución innovadora. El $47 \%$ manifiesta que su emprendimiento tiene impactos positivos en el ambiente y el $41 \%$ en lo social, ya sea porque contribuye a la accesibilidad de personas en situación de discapacidad, o bien, porque contribuye con la inclusión de personas en situación de vulnerabilidad socioeconómica.

\section{Tabla 5. Impacto de los emprendimien- tos tecnológicos}

\begin{tabular}{lc}
\hline Categorías & $\mathbf{N}^{\circ}$ \\
\hline Facturación anual & U\$S 5 millones \\
Empleo & 1371 \\
Solución disruptiva & $54 \%$ \\
Mejora medioambiental & $47 \%$ \\
Impacto social positivo & $41 \%$
\end{tabular}

Fuente: Elaboración propia en base a Startup Córdoba Monitor

Por último, como se mencionó anteriormente, los emprendimientos tecnológicos generan importantes externalidades positivas sobre la estructura económica de un territorio, puesto que tienen el potencial de dotar de nuevas tecnologías e innovaciones al resto de los sectores productivos. En la Ciudad de Córdoba, este es un aspecto importante si se tiene en cuenta que más del $50 \%$ de los emprendimientos tecnológicos desarrollan específicamente soluciones para empresas, presentando un modelo de negocio B2B 
(business to business). Las vinculaciones emprendimiento-empresa promueven la modernización de los procesos productivos de las empresas, como así también permiten la diversificación en la cartera de productos, con el potencial de capitalizar canales de ventas ya desarrollados e incrementar los ingresos.

\section{Comparación de los emprendimientos tecnológicos cordobeses con otros ecosis- temas}

La Ciudad de Córdoba es reconocida regionalmente por su ecosistema emprendedor tecnológico. Su aparato productivo, junto con su entramado institucional compuesto por incubadoras, aceleradoras y fundaciones, favorecen la proliferación de una gran cantidad de emprendimientos tecnológicos con alto potencial de crecimiento e impacto.

Según uno de los informes más completos a nivel global, el "Global Startup Index Report 2021" que publica anualmente la organización "Startup Blink" de Israel, en el que establece un ranking entre los ecosistemas de emprendimientos tecnológicos de 100 países y 1.000 ciudades de todo el mundo, el ecosistema de emprendimientos tecnológicos de Argentina se encuentra entre los líderes regionales en innovación, clasificado en el puesto número 3 de Latinoamérica. A nivel mundial se posiciona número 39 (bajando 1 puesto respecto al 2020). Más aún, hay 5 ciudades clasificadas en Argentina entre las mil mejores en el mundo: la Ciudad de Buenos Aires encabeza el listado en el puesto 60 a nivel mundial; le siguen la Ciudad de Córdoba en el puesto 297, luego Mendoza (516), Rosario (872) y Sunchales (975). La Ciudad de Córdoba muestra un impulso positivo el último año con un aumento de 22 puestos respecto al año 2020. Además, ocupa el puesto número 2 en Argentina y el número 13 en Latinoamérica. El mapa global de Startup Blink también destaca en la Ciudad de Córdoba la existencia de 132 emprendimientos tecnológicos, 2 aceleradoras y 16 organizaciones de apoyo.

En este artículo, para realizar una comparación con otros ecosistemas, se han elegido los ecosistemas de emprendimientos tec- nológicos de España y Alemania debido al relevamiento y publicación de datos que se realizan en dichos países, al igual que en la Ciudad de Córdoba. En este punto, cabe remarcar la escasez de datos existente a nivel ciudades en Latinoamérica en términos de emprendimientos tecnológicos.

En la Tabla 6 se presentan algunos puntos de comparación de los ecosistemas mencionados para el año 2020. En la primera categoría, se muestra la cantidad promedio de socios fundadores por emprendimiento en el que Córdoba muestra un valor de 3,2 integrantes, mientras que España tiene un promedio de 3 integrantes y los emprendimientos tecnológicos alemanes son las que se conforman con un menor número promedio de 2,4 integrantes.

Respecto a la edad de los fundadores se encuentra que en los tres ecosistemas tienen fundadores con edad promedio de 34 años. Sin embargo, se observa una brecha de género que es compartida en los tres casos cuando se analiza el porcentaje de mujeres fundadoras. Si bien la Ciudad de Córdoba tiene el valor más desfavorable con $14 \%$ de mujeres fundadoras, no se encuentra tan alejado de España y Alemania, donde los porcentajes son del $18 \%$ y $15,7 \%$ respectivamente.

En cuanto al número de empleados, los emprendimientos tecnológicos de Alemania presentan el mayor número promedio con 13,3 empleados. Luego, le sigue Córdoba con 9,3 y, por último, España con 6,4. Cabe aclarar que, al ser un promedio, los valores pueden tener un sesgo cuando existe un emprendimiento con un número importante de empleados. Más allá de los promedios, la mayoría de los emprendimientos tecnológicos tienen entre 1 y 3 empleados en los tres casos analizados.

Respecto al financiamiento, el $75 \%$ de los emprendimientos tecnológicos de Córdoba accedieron a financiamiento, principalmente fondos públicos, mientras que en España y Alemania el porcentaje se reduce al $53 \%$ y $55,3 \%$ respectivamente, pero donde principalmente son fondos privados. Si bien la 
estadística no muestra el valor absoluto del financiamiento obtenido, de todos modos, este indicador demuestra la existencia de programas de apoyo económicos para emprendimientos en la Ciudad de Córdoba.

Las tecnologías más utilizadas por los emprendimientos tecnológicos están bien diversificadas en los tres territorios, siendo las soluciones móviles, la computación en la nube, la inteligencia artificial y el internet de las cosas las principales.

Por último, en cuanto a la composición por rubros, aparecen algunas diferencias, aunque en los tres casos existe una gran diversificación. En el caso de Córdoba los emprendimientos de software factory y el comercio electrónico son los que tienen mayor parti- cipación, seguidos por los emprendimientos tecnológicos de salud, educación y finanzas. En España, los cinco sectores con mayor participación son los emprendimientos de finanzas, de bienestar, educación, software factory y los orientados al impacto social. Finalmente, en Alemania, la composición de los de mayor participación es distinta, siendo software factory, alimentación, salud, automoción y logística y recursos humanos los cinco primeros.

Comparando con otros ecosistemas internacionales, específicamente con el español y alemán, no se encuentran grandes diferencias en los perfiles de los emprendimientos. Ello obedece a que se trata de organizaciones de perfil global que funcionan con modelos de negocios exponenciales apalancados en las nuevas tecnologías.

Tabla 6. Cuadro comparativo con otros ecosistemas

\begin{tabular}{|c|c|c|c|}
\hline Categorías & Córdoba & España & Alemania \\
\hline Socios Fundadores & 3,2 & 3 & 2,4 \\
\hline Edad promedio de los fundadores & 34 & 34 & 34 \\
\hline$\%$ de mujeres fundadoras & $14 \%$ & $18 \%$ & $15,7 \%$ \\
\hline Empleados por emprendimiento & 9,3 & 6,4 & 13,3 \\
\hline $\begin{array}{l}\text { Nivel de estudios universitarios o } \\
\text { superior }\end{array}$ & $78,3 \%$ & $95 \%$ & $81,7 \%$ \\
\hline Acceso a financiamiento & $75 \%$ & $53 \%$ & $55,3 \%$ \\
\hline Principal tecnología & $\begin{array}{c}\text { Soluciones } \\
\text { móviles } \\
(48 \%) \\
\text { Big Data } \\
(31 \%) \\
\text { Inteligencia } \\
\text { artificial } \\
(28 \%)\end{array}$ & $\begin{array}{c}\text { Soluciones } \\
\text { Móviles } \\
(31 \%) \\
\text { Inteligencia } \\
\text { artificial } \\
(29 \%) \\
\text { Blockchain } \\
(26 \%)\end{array}$ & $\begin{array}{c}\text { Inteligencia } \\
\text { artificial } \\
(40 \%) \\
\text { Realidad virtual } \\
(24,4 \%) \\
\text { Blockchain } \\
(22,1 \%)\end{array}$ \\
\hline Verticales principales & $\begin{array}{c}\text { Software } \\
\text { factory }(19,8 \%) \\
\text { E-commerce } \\
(19,8 \%) \\
\text { E-health } \\
(16,2 \%) \\
\text { Edtech } \\
(13,2 \%) \\
\text { Fintech } \\
(10,8 \%)\end{array}$ & $\begin{array}{c}\text { Fintech } \\
(21 \%) \\
\text { Healthcare } \\
(19 \%) \\
\text { Educación } \\
(14 \%) \\
\text { Software Fac- } \\
\text { tory } \\
(13 \%) \\
\text { Impacto Social } \\
(12 \%)\end{array}$ & $\begin{array}{c}\text { Software } \\
\text { factory }(30,2 \%) \\
\text { Alimentación } \\
(10,6 \%) \\
\text { Salud } \\
(8,5 \%) \\
\text { Automoción y } \\
\text { logística }(6,7 \%) \\
\text { Recursos Hu- } \\
\text { manos } \\
(4,2 \%)\end{array}$ \\
\hline
\end{tabular}




\section{Conclusiones}

Los emprendimientos tecnológicos constituyen eslabones fundamentales en el desarrollo económico y social. Por lo que ha comenzado a fomentarse la creación y consolidación de ecosistemas de apoyo al emprendimiento en el sector tecnológico impactando en aspectos económicos, culturales y sociales.

La introducción de productos y servicios intensivos en conocimiento fomenta la creación de empleo de alto valor agregado, genera mejoras en la experiencia usuario, contribuye a la accesibilidad y por consiguiente aumenta la calidad de vida de los ciudadanos. De igual forma, el progreso tecnológico afecta al desarrollo social y económico en cuanto al efecto innovador que puede implicar para muchas otras industrias.

El presente artículo intentó dar cuenta del perfil del ecosistema de emprendimientos tecnológicos de la Ciudad de Córdoba, remarcando la importancia del mismo para la economía local. Como conclusión, se extrae que la Ciudad de Córdoba cuenta con un entramado institucional apto para la proliferación de emprendimientos tecnológicos. Además, se observa un significativo número de ellos ya funcionando y creciendo en la ciudad.

Como principales aspectos a mejorar del ecosistema local puede señalarse la necesidad de generar un crecimiento del capital de riesgo, una mayor incorporación de mujeres al sector tecnológico, incrementar los niveles de facturación de los emprendimientos y favorecer la internacionalización de estos.

\section{REFERENCIAS BIBLIOGRÁFICAS}

- Birch, David G.W. (1987), Job Creation in America: How Our Smallest Companies Put the Most People to Work University of Illinois at Urbana-Champaign's Academy for Entrepreneurial Leadership Historical Research Reference in Entrepreneurship, Available at SSRN: https://ssrn.com/abstract=1496185

- Castillo-Vergara M. (2020) “La teoría de las $\mathrm{N}$-hélices en los tiempos de hoy" J. Technolo- gy Management and Innovation 2020. Volume 15, Issue 3.

- Crisafulli, L. y Parisi, D. (2020): El ecosistema emprendedor tecnológico de Córdoba, Municipalidad de Córdoba.

- Crisafulli, L. y Parisi D. (2020): Emprendimientos tecnológicos en la ciudad de Córdoba, Municipalidad de Córdoba.

- Ewing Marion Kauffmann Foundation (2014) The importance of young firms for economics growth. Entrepreneurship Policy Digest, September 25.

-Etzkowitz, H., Leydesdorff, L. (2000). The Dynamics of Innovation: From National Systems and 'Mode 2' to a Triple Helix of UniversityIndustry-Government Relations. Research Policy, 29(2), 109-123.

- Isenberg, D. (2011). The entrepreneur ecosystem strategy as a new paradigm for economic policy: Principles for cultivating entrepreneurship. Babson Global, s/n.

- Kantis, H., Federico, J., \& Ibarra, S. (2016). Condiciones sistémicas para el emprendimiento dinámico 2016. Programa de Desarrollo Emprendedor PRODEM.

- Kantis, H., Angelelli, P. (2020) Emprendimientos de base científico-tecnológica en América Latina: Importancia, desafíos y recomendaciones para el futuro, BID, mimeo

- OCDE (2013) Startup América Latina: Promoviendo la Innovación en la Región, OECD Development Centre Studies, Paris, France.

- OCDE (2016) Startup América Latina: Construyendo un Futuro Innovador. OCDE Development Centre Studies, Paris, France.

- Patiño, D. y García de Leon, V. (2018): “4 elementos necesarios para que la economía crezca". Expansión. Volumen 21.

- Schumpeter, J. A. (1942). Capitalismo, socialismo y democracia. Barcelona, Orbis, 1983.

- Solow, R. (1957). Technical change and the aggregate production function. Review of Economics and Statistics, 39, 312-320.

- Varga, A., Schalk, H.J. (2004). Knowledge spillovers, agglomeration and macroeconomic growth: An empirical approach. Regional Studies, 38(8), 977-989.

- West P. G., Bamford C. (2005) Creating a Technology-Based Entrepreneurial Economy: A Resource Based Theory Perspective. Journal of Technology Transfer, 30(4), 433-451. 